\title{
CARDIAC VAGAL TONE AS A PSYCHOPHYSIOLOGICAL INDEX OF FRONTO-LIMBIC DYSFUNCTION DURING EMOTION REGULATION IN BORDERLINE PERSONALITY DISORDER.
}

Wainsztein A. ${ }^{1-3}$, Goldberg X. ${ }^{4}$, Alvarez Mercé R. ${ }^{1}$, Camacho V. ${ }^{1,5}$, Vulcano M. ${ }^{2}$, Abulafia C. ${ }^{1-3,5}$, Vigo D. ${ }^{3,5}$, Villarreal M. ${ }^{1,5}$, Cardoner N. ${ }^{4}$, Nemeroff C. ${ }^{6}$, Castro M. ${ }^{1,5}$, Soriano-Mas C. ${ }^{7}$, Guinjoan S. ${ }^{1,2,5}$.

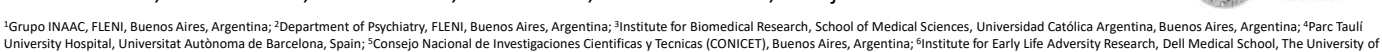

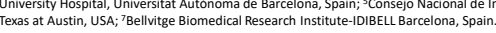

\section{Introduction}

Autonomic network overlaps with brain regions that command executive, cognitive, behavioral and emotional processes. Thus, heart rate variability (HRV) has been studied during resting states in emotional disorders. We examined functional neuroimaging and autonomic correlates during emotion regulation in Borderline Personality Disorder (BPD) and Healthy Controls (HC).

\section{Methods}

Thirty-nine right-handed subjects $(H C, n=20 ; B P D, n=19)$ matched for age and gender (Table 1), were trained on emotion regulation strategies (e.g. cognitive reapraissal) and performed an emotional task (OBSERVE, MAINTAIN and REGULATE, Fig 1). We used a 3T-GE MRI and simultaneous BIOPAC-HRV acquisition. BOLD signal was analyzed using SPM12. HRV-time-domain variables ( $m m R$, SDNN, RMSSD) were calculated using Kubios software. Self-reported questionnaires on Adverse Childhood Experiences (ACE) and difficulties in emotion regulation (DERS) were assessed.

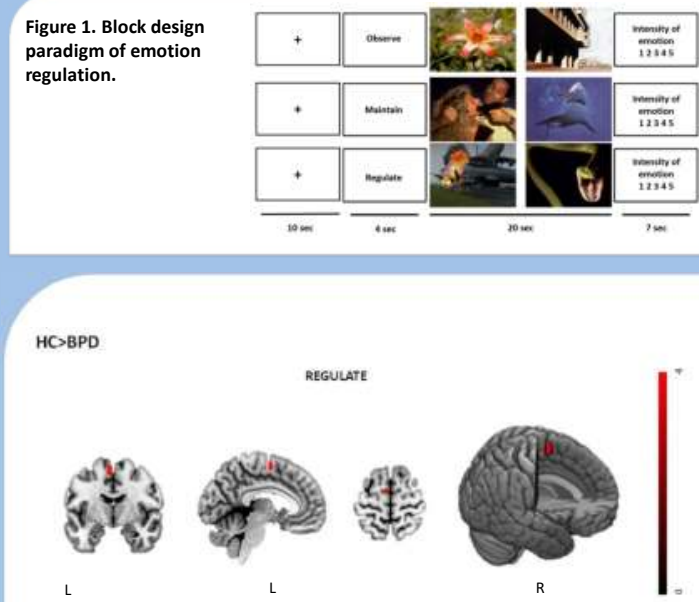

Figure 2. Brain activation during REGULATE task in $\mathrm{HC}$ vs. BPD (HC>BPD). HC display activation of left supplementary motor area (MNI coordinates: $x=-6, y=-6, z=64$; $K=67 ; Z=3.42$ ) in contrast to patients. All results FWE corrected $p<0.05$ across small volume correction. L-SMA: Left Supplementary Motor Area.

Table 1. Clinical and Demographic characteristics of study groups.
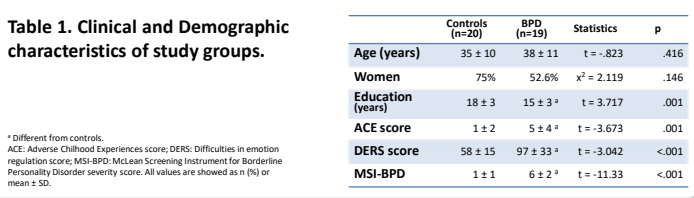

\section{Results}

Deficit of activation in the left supplementary motor area (L-SMA; $p=0.024$ ) was found during REGULATE condition in BPD patients (Fig 2). During this task, $\mathrm{HC}$ showed a positive correlation between RMSSD with brain activation in L-SMA ( $p=0.035)$, right superior frontal gyrus (R_SFG; $p=0.017$ ) and left dorsolateral prefrontal cortex areas (L_DLPFC; $p=0.017$, Fig 2). Left amygdala, hippocampus and anterior cingulate were negatively correlated with RMSSD in the BPD group during REGULATE task (L_AMG: $p=0.018$; L_HIP: $p=0.021$; L ACC: $p=0.018$ respectively, Fig 3); neglect and household dysfunction ACE subscores had significant influence in controlling for the latter.

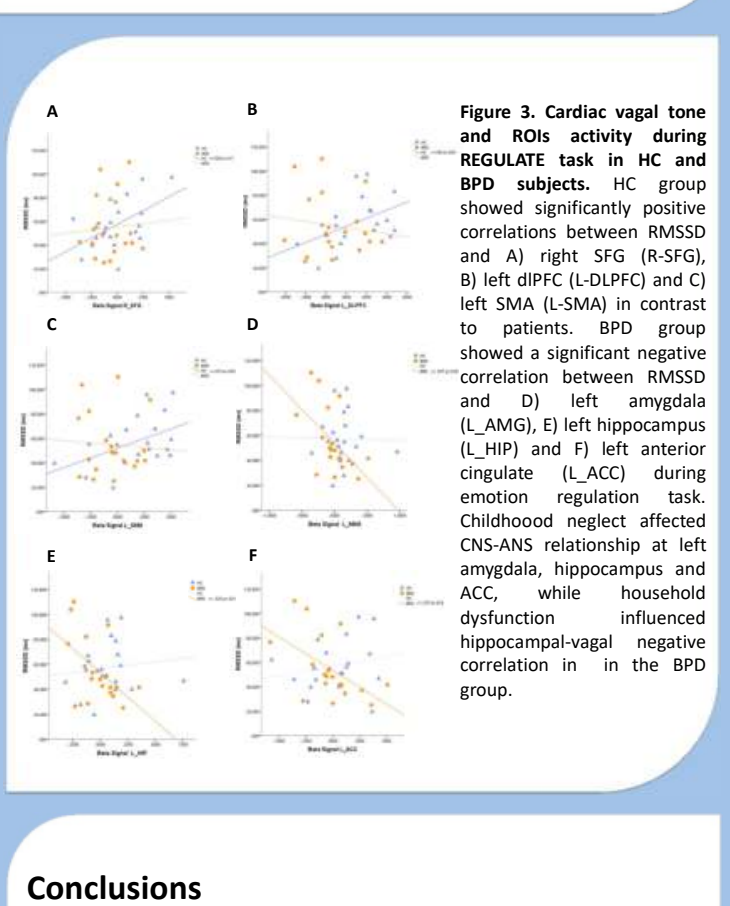

Activation deficit of L-SMA suggests an abnormal pattern of conscious emotion regulation process in BPD patients compared to healthy subjects. Lower cardiac vagal tone during regulation of emotional stimuli could represent a psychophysiological biomarker of inadequate frontal-limbic interaction and hence, a less adaptive emotion regulation capacity in BPD individuals. Childhood traumatic events modulate this CNS-ANS interrelations. Future research directions attempt to explore the profound effects of early life stress on brain development and emotional behavior in this population. 